\title{
Gini, el cambio en la desigualdad y sus componentes
}

\section{Fernando Cortés y Rosa María Rubalcava}

A pesar de que el índice de Gini está diseñado para medir ei grado de concentración de una variable, frecuentemente se utiliza para analizar el cambio experimentado por la desigualdad en la distribución, en el tiempo o en el espacio.

En los estudios en que interesa la evolución de la desigualdad, el cambio en los valores del índice puede deberse no sólo a modificaciones en la distribución de la variable, sino también a cambios en los tamaños relativos de los grupos entre los cuales se distribuye.

Este trabajo propone una descomposición del índice de Gini que permite separar su variación en tres componentes: (i) el efecto de los tamaños de los grupos, (ii) el efecto de las participaciones relativas de los grupos y (iii) un efecto que recoge los componentes no separables de los tamaños y de las participaciones relativas.

\section{Presentación del problema}

El índice de concentración de $\mathrm{Gini}^{1}$ fue propuesto inicialmente para medir la desigualdad en la distribución de un total entre un conjunto de individuos. ${ }^{2}$ De aquí que para aplicarse se requiera, en términos estrictos, que: (i) el total a distribuir se agote una vez que se repartió, (ii) interese medir el grado de la desigualdad ${ }^{3}$ y (iii) que los beneficiarios de la repartición sean individuos. ${ }^{4}$

La tercera de estas condiciones lleva a la discusión del grado de subestimación en que se incurre al medir la desigualdad con datos agrupados en lugar de usar las observaciones individuales. En otra parte ${ }^{5}$ hemos presentado argumentos que relativizan esta preocupación según el tipo de referentes empíricos que define la teoría. En todo caso, no es usual tener acceso a los datos originales por lo que el investigador se ve limitado a utilizar las distribuciones de frecuencias publicadas por los organismos encargados de la producción de la información. Habitualmente, estas distribuciones de frecuencias no coinciden con las que requiere el investigador ya sea porque no hay una correspondencia entre las definiciones ad-

\footnotetext{
${ }^{1}$ Corrado Gini, Curso de Estadística, Editorial Labor, Barcelona, 1935, pp. 163 a 177.

${ }^{2}$ Empleamos la palabra individuo en su sentido estadístico de entidad individual.

${ }^{3}$ Debe diferenciarse entre el nivel y el grado de la desigualdad. Un tratamiento simple de este tópico se encuentra en Adalberto García Rocha, La desigualdad económica, El Colegio de México, México, en prensa, cap. 1, y en Fernando Cortés y Rosa María Rubalcava, Técnicas estadísticas para el estudio de la desigualdad social, El Colegio de México, México, 1982, cap. 1.

${ }^{4}$ Un estudio de las propiedades matemáticas del coeficiente de Gini se encuentra en Dominique Thon, "An axiomatization of the Gini coefficient", Mathematical Social Sciences, 2 (1982), pp. 131 a 134, North Holland Publishing Co.

5 Véase, Cortés, Fernando y Rosa María Rubalcava, Técnicas estadísticas..., op. cit, pp. 141 a 147.
} 
ministrativas y teóricas, o porque se involucran diferentes niveles de agregación.

La interpretación del valor de un coeficiente de concentración con un recorrido definido implica una doble comparación. Una estadística, consiste en localizar el valor en cuestión en una escala (normalmente con extremos cero y uno) que permite graduar la "gravedad" de la desigualdad (por consideraciones de carácter lógico se acostumbra definir el cero como ausencia de concentración, es decir, como equidistribución y el uno como concentración máxima). Sin embargo, es habitual que esta escala (con interpretación clara asignada únicamente a los valores extremos) no provea al investigador de interpretaciones satisfactorias. Una mejor comprensión del significado que puede atribuirse a una medición particular de desigualdad requiere que se introduzca información factual explícita o implícitamente mediante comparaciones en el espacio, en el tiempo, o en una combinación de ambos, ${ }^{6}$ tomando como base de la comparación otro contexto o el mismo. Saber que el grado de concentración de la tierra en un país, en un tiempo dado, es $\mathbf{0 . 8 5}$, sólo nos permite afirmar que se trata de un valor más próximo a uno que a cero. No derivamos mayor información, a menos que se use además el patrón del grado de concentración de la tierra en un momento anterior o posterior en el mismo país o en otro que se haya decidido utilizar como punto de comparación.

Las variaciones que experimenta el grado de la desigualdad a través del tiempo en un sistema social dado resultan de dos procesos. Uno que afecta los parámetros de la distribución y otro que surge de la evolución temporal de los tamaños relativos de los grupos sociales entre los cuales se reparte el total. Los factores de que dependen las dinámicas de estos procesos suelen ser diferentes, por lo que la medición del aporte que realiza cada uno de ellos es un paso necesario para alcanzar una explicación satisfactoria de la evolución temporal del grado de desigualdad. De aquí nuestro interés por disponer de una descomposición de la variación experimentada por el índice de Gini entre dos o más momentos.?

Con el propósito de ilustrar las ideas expuestas y, a la vez, tener referentes concretos que permitan una mejor comprensión de los resultados

${ }^{6} \mathrm{Si}$ bien la descomposición se puede aplicar a comparaciones referidas a dos lugares distintos en un mismo tiempo, o a una combinación de espacio y tiempo (que requiere de una extensión trivial de las fórmulas que se presentan más adelante), este texto enfatiza el análisis cronológico de la desigualdad.

7 Cuando las curvas de Lorenz se cortan, la comparación del grado de desigualdad es inconclusiva porque depende del índice de desigualdad que se utilice. Sin embargo, si ya se decidió por un índice particular (como es el caso que tratamos en este trabajo), no hay dificultades en la comparación, debido a que las ponderaciones de las diferencias que componen el índice son constantes. Para mayores detalles puede consultarse, por ejemplo, A.B. Atkinsons, The Eccnomics of Inequolity, Clarendon Press, Oxford, 1975, p. 47, y Adalberto García Rocha, La desigualdad económica, El Colegio de México, México, 1986, cap. III. 
algebraicos que se exponen en la sección que sigue, permítasenos considerar tres ejemplos:

(i) El cambio en el grado de concentración de la tierra es el resultado, por una parte, del proceso de fraccionamiento de la propiedad agraria que en los campesinos pobres se relaciona con la tasa de reproducción demográfica y, por otra, del proceso, que refuerza al anterior, de concen tración de la tierra en manos de los empresarios privados. (La explicación de esto dependerá de la teoría con que se enfrente al fenómeno: puede ser una consecuencia lógica de la búsqueda de la ganancia - es una ley del capitalismo-, o deberse a la existencia de tamaños óptimos para las explotaciones o a características de la personalidad campesina y patronal, etc.) Otra fuente de variación podría ser la intervención estatal, que a través de medidas redistributivas, modificaría simultáneamente los tamaños relativos de los grupos y las proporciones de tierra que les corresponden.

(ii) El cambio en la distribución del ingreso a través del tiempo podría deberse a tasas diferenciales de crecimiento demográfico de los estratos o clases sociales (según el tipo de estudio), a alteraciones en los parámetros que determinan las participaciones de los grupos sociales en el ingreso total (ya sea del ingreso personal o del ingreso familiar) o a modificaciones que afecten simultáneamente los tamaños relativos de los estratos y la parte del total que recibe cada uno de ellos, como sería, por ejemplo, la resultante de una política redistributiva que ampliara los sectores medios.

(iii) La variación que experimenta la distribución del presupuesto fiscal entre las Secretarías de Estado depende fundamentalmente de los objetivos económico-sociales que se propongan las autoridades, los que se traducen en criterios de asignación de recursos. Como en este caso los tamaños relativos de los "beneficiados" son más o menos estables, el cambio en la desigualdad quedará explicado, básicamente, por la política económico-social realmente aplicada.

En aquellos casos en que no se modifican los tamaños relativos (o excluyentes) o las participaciones relativas, desaparece el efecto conjunto. Por consiguiente, las variaciones experimentadas por el grado de la desigualdad a través del tiempo estarán determinadas sólo por los factores que mueven uno u otro proceso.

El control de los tamaños relativos de los intervalos de clase de las distribuciones de frecuencias simplifica el análisis estadístico del problema que nos interesa por cuanto el cambio en la desigualdad dependerá únicamente de las modificaciones en las participaciones relativas. Este procedimiento, adecuado desde la perspectiva de la estadística, puede entrar en contradicciones o no representar adecuadamente manifestaciones empíricas de categorías conceptuales, o bien no mostrar la situación de grupos sociales existentes en la sociedad. Un claro ejemplo del tipo de situación que hemos señalado es el del estudio del cambio en la distribución 
del ingreso por "deciles", ${ }^{8}$ hasta donde sabemos, ésta es una categoría estadística que no tiene relación con ningún concepto de la teoría económica.

Por otra parte, es sabido que los científicos sociales raras veces acceden a la información primaria, de manera que habitualmente tienen que conformarse con construir las mejores aproximaciones a los conceptos teóricos a partir de las publicaciones oficiales. ${ }^{9} \mathrm{Si}$ los tamaños relativos de los intervalos de clase de dos o más distribuciones de frecuencias referidas a tiempos o espacios distintos son los mismos, podemos descomponer el cambio del índice de Gini en los efectos "propagación" y "ventaja". ${ }^{10}$ El estudio del cambio en el grado de la concentración de una variable a través del tiempo se complica en el caso de que varíen tanto los tamaños relativos cuanto las participaciones de los estratos.

II. Una descomposición del cambio en el índice de Gini

Una de la varias fórmulas que sirve para calcular el índice de Gini para datos agrupados es:

$$
\mathrm{G}=1-\sum_{i=1}^{m} p_{i}\left(Q_{i}+Q_{i-1}\right)
$$

donde:

$\mathbf{p}_{i}$ representa el tamaño relativo del intervalo de clase $\mathbf{i}$,

$Q_{i}$ y $Q_{j-1}$ simbolizan las participaciones relativas acumuladas en la variable de los estratos $i$ e (i-1), y

$\mathrm{m}$, es el número de intervalos de clase.

El desarrollo de la suma y la agrupación conveniente de términos genera la ecuación:

$$
G=1-\sum_{i=1}^{m} p_{i} q_{i}-2 \sum_{i=2}^{m} p_{i}\left(\sum_{i=1}^{i-1} q_{i}\right)
$$

${ }^{8}$ Es decir, por grupos de $10 \%$ de perceptores, previa ordenación de los mismos.

${ }^{\theta}$ Este tema ha sido objeto de preocupación constante de los científicos sociales de América Latina, lo que se ha traducido en una vasta producción, originada en gran parte en el seno de Clacso. Consúltese, por ejemplo, Susana Torrado [comp.], Los censos de población y de vivienda en la década de 1980 en América Latina, Clacso, Comisión de población y desarrollo, Grupo de trabajo sobre información socio-demográfica, Buenos Aires, 1981. Un trabajo que intenta construir referentes empíricos de conceptos marxistas es el de Emilio de Ipola, et al, Teoría y método para el estudio de la estructura de clases social (un análisis concreta: Chile 1970J, Flacso, Proelce, Santiago, 1976. Trabajos interesantes son los de Ian Miles y John Irving, "The critique of official statistics" y el del Government statistician's colective, "How official statistics are produced: views from the insight", ambos en John Irving, Ian Miles y Jeff Evans (eds.), Demistifying social statistic, Pluto Press, London, 1979.

${ }^{10}$ Ver Fernando Cortés, y Rosa María Rubalcava, "Análisis del cambio en la concentración a través del coeficiente de Gini", en Demografía y Economía, val. XVII, núm. 4 [56), 1983. 
Llamemos $\delta \mathrm{p}_{i}$ al cambio en el tamaño relativo del estrato $\mathrm{i}$ entre los tiempos $t$ y $(t+1)$. Las variaciones experimentadas en $p_{i}$ al mantener constante las participaciones en la variable inducirán una modificación del indice de Gini $\left(\delta G_{p}\right)$ :

$$
\text { (1) } \delta G_{p}=-\sum_{i=1}^{m}\left(\delta p_{j}\right) q_{i}-2 \sum_{i=2}^{m} \delta p_{i}\left(\sum_{j=1}^{j-1} q_{j}\right)
$$

Los cambios relativos en las participaciones manteniendo constantes los tamaños relativos originan un cambio en Gini $\delta G_{q}$ que origina la ecuación:

$$
\delta G_{q}=-\sum_{j=1}^{m} p_{i}\left(\delta q_{i}\right)-2 \sum_{j=2}^{m} p_{i}\left(\sum_{j=1}^{j-1} \delta q_{j}\right)
$$

Si se alteran simultáneamente los tamaños y las participaciones relativas $\left(\delta p_{i}\right.$ y d $\left.q_{i}\right)$ se tiene la ecuación:

$$
\text { (3) } \delta G_{p q}=-\sum_{i=1}^{m}\left(\delta p_{i}\right)\left(\delta q_{i}\right)-2 \sum_{i=2}^{m} \delta p_{i}\left(\sum_{j=1}^{j-1} \delta q_{j}\right)
$$

Por medio de simples operaciones algebraicas se establece que:

$$
\text { (4) } \delta G=\delta G_{p}+\delta G_{q}+\delta G_{p q}
$$

donde $\delta G$, simboliza el cambio en el índice de Gini entre $t$ y $(t+1)$, y la ecuación (4) muestra los tres componentes que lo constituyen.

Sabemos, por los desarrollos presentados, que $\delta G_{p}$ representa aquella parte del cambio en el grado de desigualdad debida a las variaciones en los tamaños relativos de los estratos manteniendo constantes sus correspondientes participaciones. Esta situación sería observacionalmente posible si (i) las tasas de crecimiento de los intervalos de clase fuesen diferenciales, de modo que los estratos de crecimiento más rápido sufrieran un empobrecimiento relativo (recuérdese que las participaciones relativas son constantes) y que los de crecimiento más lento (y con mayor razón los que disminuyen sus tamaños relativos) mejoraran su posición. Estos movimientos se reflejarían en las razones de ventajas ${ }^{11}\left(q_{i} / p_{i}\right.$ suponiendo que $\mathrm{q}_{i}$ no se ha modificado), las que en el primer caso disminuirían y en el segundo aumentarían; (ii) la descripción presentada en (i) supone que los cambios en las razones de ventaja no son demasiado violentas, de modo que no se producen flujos de casos entre intervalos de clase; sin embargo, hay que considerar que los tamaños relativos pueden variar a consecuencia de transferencias de observaciones entre estratos. Para cumplir con la condición de no modificación en las participaciones relativas, deberá

11 Ver Heyward, Alker, El uso de las matemáticas en el análisis político, Amorrortu, Buenos Aires, 1975. 
suponerse que este término sólo da cuenta de los flujos que se neutralizan; (iii) el caso más frecuente en las aplicaciones empíricas será, probablemente, el de una combinación de las señaladas. La interpretación del valor de $\delta G_{p}$ en un aplicación particular dará mayor peso a la interpretación (i) o a la (ii), según las características específicas del problema estudiado. Por ejemplo, considerando si los intervalos de clase se reproducen o no (y, en caso afirmativo las tasas históricas con que lo hacen), o bien evaluando las probabilidades de transición entre estratos.

El término $\delta G_{q}$, cuantifica la contribución de las variaciones en las participaciones relativas de los estratos a las diferencias entre $t$ y $(t+1)$, suponiendo que los tamaños relativos no se alteran. En este caso también distinguiremos, por razones analíticas, tres casos: (i) que al cambiar las participaciones relativas de los estratos habrá algunos beneficiados y otros perjudicados (lo que se podría observar en las razones de ventaja, bajo el supuesto de que los $p_{j}$ se mantienen], pero que son de tal magnitud que no hay tránsitos interestratos; (ii) que aun cuando haya transiciones, éstas se compensan de manera que los tamaños relativos permanecen inalterados; (iii) que lo más probable es que las situaciones empíricas resulten ser una combinación de lo descrito en (i) y (ii). La evaluación de la contribución relativa de las posibles fuentes dependerá, nuevamente, de información adicional que se pueda recabar en relación al caso analizado.

El último componente de la ecuación (4), $\delta G_{p q}$, mide aquella parte del cambio en el índice de Gini que resulta de los movimientos conjuntos en los tamaños y de las participaciones relativas. Por lo tanto, una parte de la variación en el valor del índice de Gini resulta de las combinaciones no compensadas de redistribuciones y de tránsitos.

Ahora bien, si entre $t y(t+1)$, se han mantenido constantes los tamaños relativos, es decir, si $\delta G_{p}=0$, la ecuación (4) se reduce a:

$$
\delta G=\delta G_{q} \text {, }
$$

y por consiguiente, el cambio en el índice de Gini se limita a reflejar la redistribución del valor total de la variable. La condición $\delta G_{p}=0$ puede ser producto de un hecho o de un procedimiento estadístico de control de variable. Esta última situación es la que se presenta, en particular, cuando se analizan las tendencias en la distribución del ingreso por deciles $\mathrm{y}$, en general, cada vez que se construyen distribuciones de frecuencias con el criterio de mantener los tamaños relativos de los estratos.

Si en el periodo considerado no se han alterado las participaciones relativas de los intervalos de clase, es decir, si $\delta G_{q}=0$ entonces tenemos que (4) asume la forma:

$$
\delta G=\delta G_{p},
$$

esta igualdad nos señala que el valor del índice de Gini se modificará a 
consecuencia de variaciones en los tamaños relativos. Esto quiere decir que si las clases se reproducen a tasas diferenciales o hay transiciones de observaciones entre los intervalos, sujetas a la restricción de que no alteren las participaciones relativas, de los estratos, entonces se observará un $\delta \mathrm{G}$ diferente de cero. Decíamos que si se acopia información adicional es posible evaluar el peso relativo de estos dos componentes. En el caso de que se detecte que el proceso dominante es la reproducción de los estratos, se concluye que el índice de Gini mostraría un cambio en la desigualdad, debido, por ejemplo, a la dinámica demográfica diferencial (como sería el caso de la distribución del ingreso o de la tierra). Si la mayor importancia la tuviesen los saltos de intervalos de clase entonces el $\delta \mathrm{G}$ se debería a procesos de movilidad social.

Las aplicaciones habituales del índice de Gini se realizan sobre distribuciones de frecuencias en que los intervalos de clase se definen sobre la variable que es objeto de la repartición. Por ejemplo, la distribución de la tierra entre agricultores usa habitualmente como variable de clasificación el área de los predios, originándose de este modo categorías como microfincas, fincas familiares, etc. Lo mismo acontece con la distribución del ingreso. Pero hay casos en que interesa estudiar la distribución de una variable en relación a otra, como por ejemplo, la repartición del producto geográfico entre la población de los estados de la República, o de los estudiantes universitarios clasificados según la ocupación del padre en la PEA distribuida por ocupación. El índice de Gini está específicamente diseñado para tratar el primer tipo de distribuciones de frecuencias; sin embargo, cuando interesa estudiar la distribución de una variable en relación a otra es necesario que ambas estén definidas sobre la misma unidad ${ }^{12}$ (es decir, que las observaciones sean pares ordenados de las unidades).

Interesa diferenciar entre ambos casos, porque es distinta la "sensibilidad estadística" de los movimientos interestrato ante modificaciones en la distribución de la variable. En el primer tipo de distribución de frecuencias, la redistribución hará que algunas unidades cambien de intervalo tan sólo por el hecho de recibir más (o menos según sea el caso). De modo que cambios en las participaciones relativas tenderán a inducir modificaciones en los tamaños relativos de los intervalos de clase. En el segundo tipo de distribución, la clasificación de las observaciones tendrá poco que ver con redistribuciones. Como consecuencia de lo anterior, debería mos esperar que el tamaño relativo del término $\delta \mathrm{G}_{\mathrm{pq}}$ sea tendencialmente mayor en el primer tipo de distribución de frecuencias que en el segundo.

12 Un tratamiento teórico de este tema se encuentra en Marc Barbut, "Sur quelques proprietes elementaires des fonctions de concentration de C. Gini", Math. Sci. Hum., 22 anne, núm. 88, 1984, pp. 5 a 19. Para una aplicación, ver Marc Barbut, "Note sur quelques indicateurs globaux de l'inegalité: C. Gini, V. Pareto, P. Lévy", Revue francaise de sociologie, XXV, 1984, pp. 609 a 622 . 
La ecuación (4) sirve para describir el cambio del índice de Gini a través del tiempo, así como para identificar los factores inmediatos responsables del mismo. La explicación es un asunto diferente que depende en gran medida de la conceptualización en la que se localiza el problema. El resultado al que hemos llegado muestra que habrá dificultades para explicar el cambio en la desigualdad de una variable si no se separan sus componentes, debido a que, en general, obedecen a conjuntos diferentes de determinantes (demográficos, económicos, sociales y políticos). Si $r, s, t, u, v, w, x, y, z$ denotan variables, entonces se tiene que:

$\delta G_{q}=f_{1}(\mathrm{x}, \mathrm{y}, \mathrm{u}, \ldots)$

$\delta G_{p}^{q}=f_{2}(\mathrm{r}, \mathrm{s}, \mathrm{t}, \ldots)$

$\delta G_{p q}=f_{3}(v, w, t, z, u, \ldots)$ si la distribución es del primer tipo y,

$\delta G_{p q}=f_{4}(w, t, z, u, \ldots)$ si es del segundo.

La diferencia entre éstas dos últimas ecuaciones es que $f_{3}$ incorpora una variable adicional $v$, que representa la sensibilidad estadística de la distribución de frecuencias, la que deberá especificarse para cada caso concreto. En aquellos casos en que los procesos respondan a determinantes comunes, las funciones $f_{1}$ y $f_{2}$, compartirán una misma variable. Lo mismo podrá acontecer con cada una de estas funciones y, $f_{3}$ y $f_{4}$, más allá de la sensibilidad estadística $v$.

Debido a que la descomposición presentada es lineal, es posible componer el cambio en la distribución entre $t y(t+k)$ (donde $k$ es un entero), sumando las variaciones entre los tiempos intermedios. Supongamos que $k=2$ y que redefinimos la simbología agregando dos subíndices, el primero referido al tiempo contra el cual se hace la comparación y el segundo al momento actual. Así, por ejemplo, $\delta G_{t, t+1}$ representa la variación del índice de Gini en el tiempo $t+1$, en comparación con $t$. Con la nueva terminología la ecuación (4) se reescribe como:

$$
\text { (5) } \delta G_{t, t+1}=\delta G_{p, t, t+1}+\delta G_{q, t, 1+1}+\delta G_{p q, t, t+1}
$$

Al desplazar $t$ en una unidad se genera:

$$
\delta G_{t+1, t+2}=\delta G_{p, t+1, t+2}+\delta G_{q, t+1, t+2}+\delta G_{p q, t+1, t+2}
$$

Dada la linealidad de la descomposición, la suma de (5) y (6) genera:

$$
\delta G_{t, t+2}=\delta G_{p . t . t+2}+\delta G_{q . t, t+2}+\delta G_{p q . t . t+2}
$$

La generalización es a fortiori. 


\section{Dos ejemplos}

En esta sección incluimos, a modo de ilustración, un análisis del cambio en la distribución de la tierra en Guatemala entre 1950 y 1964 y entre 1964 y 1979, y en la distribución del ingreso en México entre 1970 y 1980 . Interesa mostrar, con un par de casos concretos, las bondades analíticas de la descomposición que proponemos y establecer el tipo adicional de información que nos proporciona en relación a la medida global de Gini.

Por otra parte, el hecho de que el interés de este trabajo sea metodológico-técnico nos lleva a tomar la información tal como se ha publicado, sin realizar un análisis de su consistencia. Como es habitual que la comparabilidad de los censos resulte afectada por cambios en las definiciones básicas y que para garantizarla sea necesario un trabajo que supera con creces el ámbito que hemos definido para éste,${ }^{13}$ los resultados que exponemos deben tomarse con cautela.

Por las consideraciones anteriores, el lector no debe esperar, en este artículo, explicaciones respecto a los factores que presumiblemente afectaron a los componentes del cambio ni tampoco descripciones confiables de los procesos reales.

\section{III.1 El cambio en Ia concentración de la tierra en Guatemala} en 1950, 1964 y $1979^{14}$

Con los datos del cuadro 1 hemos preparado el cuadro 2, que contiene la información básica necesaria para computar los índices de Gini así como los componentes de los cambios.

El cálculo de los coeficientes de Gini arrojó los siguientes resultados para 1950, 1964 y 1979:

$$
G_{1}=0.836 \quad G_{2}=0.785 \quad y \quad G_{3}=0.842,
$$

donde 1 simboliza 1950, 21964 y 3 1979. Por lo tanto:

$$
\delta \mathrm{G}_{1}=-0.051 \text { y } \delta \mathrm{G}_{2}=0.057
$$

La variación en el índice de Gini muestra que entre 1950 y 1964 tuvo

13 Para una evaluación del censo levantado en México en 1980 se puede consultar a Brígida García, "Dinámica ocupacional rural y urbana en el sureste de México: 1970-1980", en Demografía y Economía, vol. XVII, núm. 3[59), 1984.

14 Esta información nos fue proporcionada por Ángel Blas, alumno de la quinta promoción de Flacso-México y forma parte de los datos que utilizará en 'su tesis de maestría. 
CUADRO 1

Distribución de la tierra en Guatemala, 1950, 1964 y 1979 (cifras en miles)

\begin{tabular}{|c|c|c|c|c|c|c|}
\hline \multirow[b]{2}{*}{ Estratos } & \multicolumn{2}{|c|}{1950} & \multicolumn{2}{|c|}{1964} & \multicolumn{2}{|c|}{1979} \\
\hline & Núm. predio & Área & Núm. predio & Área & Núm. predio & $\overline{A ̄ r e a}$ \\
\hline $\overrightarrow{\text { Microfincas }}$ & 74.3 & 40.8 & 85.1 & 46.7 & 250.9 & $\overline{87.1}$ \\
\hline Fin. subfamil. & 233.8 & 720.8 & 279.8 & 869.9 & 296.7 & 881.9 \\
\hline Fin. famil. & 33.0 & 715.5 & 43.6 & 928.7 & 49.1 & 908.7 \\
\hline Multif. med. & 7.1 & 1667.9 & 8.4 & 1801.2 & 13.2 & 2566.0 \\
\hline Multif. gran. & 0.5 & 2170.5 & 0.4 & 1280.3 & 0.5 & 1367.6 \\
\hline Total & 348.7 & 5315.5 & 417.3 & 4926.8 & 610.3 & 5811.3 \\
\hline
\end{tabular}

Fuente: Dirección General de Estadística; Censos Nacionales Agropecuarios de 1950, 1964 y 1979 , Guatemala.

CUADRO 2

Tamaños y participaciones relativas y sus variaciones en el tiempo

\begin{tabular}{crrrrrrrrrr}
\hline Est. & $p_{1}$ & $q_{1}$ & $p_{2}$ & $q_{2}$ & $p_{3}$ & $q_{3}$ & $\mathrm{dp}_{1}{ }^{*}$ & $\mathrm{dq}_{1}{ }^{*}$ & $\mathrm{dp}_{2}{ }^{*}$ & $\mathrm{dq}_{2}{ }^{*}$ \\
\hline 1 & 0.213 & 0.008 & 0.204 & 0.009 & 0.411 & 0.015 & -0.009 & 0.001 & 0.207 & 0.006 \\
2 & 0.670 & 0.136 & 0.670 & 0.177 & 0.487 & 0.147 & 0.000 & 0.041 & -0.183 & -0.03 \\
& & & & & & & & & & 0 \\
3 & 0.095 & 0.135 & 0.105 & 0.188 & 0.080 & 0.184 & 0.010 & 0.053 & -0.025 & -0.04 \\
& & & & & & & & & & 0 \\
4 & 0.020 & 0.312 & 0.020 & 0.366 & 0.021 & 0.427 & 0.000 & 0.054 & 0.001 & 0.061 \\
5 & 0.002 & 0.409 & 0.001 & 0.260 & 0.001 & 0.227 & -0.001 & -0.149 & 0.000 & -0.03 \\
& & & & & & & & & & 3
\end{tabular}

* Las diferencias se calcularon tomando la cifra del año posterior menos la del anterior. Por ejemplo, $\delta p_{1}=p_{2}-p_{1}$.

lugar un proceso de desconcentración de la tierra más o menos marcado volviéndose a concentrar entre 1964 y 1979 a un punto tal que la desigualdad en 1979 arrojó un valor de Gini mayor que el de 1950.

La descomposición del cambio entre 1950 y 1964 dio como resultados:

$$
\delta G_{p, 1}=-0.004 \quad \delta G_{q, 1}=-0.048 \quad \delta G_{p q, 1}=0.001
$$

Nótese que la suma de estos tres componentes verifica la ecuación (4). Ahora bien, la descomposición muestra que la tendencia a una distribución más equitativa en la propiedad agraria entre 1950 y 1964 se debió fundamentalmente a modificaciones en las participaciones relativas (supuesto que los tamaños relativos de los estratos son constantes: $\delta G_{q, 1}, y$ que los efectos de los tamaños relativos y el conjunto no tienen importancia). Este resultado concuerda con la caída que experimentó la participación relativa del último estrato (pasó de 0.409 a 0.260 ) concomitantemente con una ganacia relativa de los restantes. Estos movimientos en los 
valores de q contrastan con la estabilidad de los tamaños relativos.

La descomposición del cambio para el periodo 1964-1979 fue:

$$
\delta G_{\mathrm{p}, 2}=0.047 \quad \delta G_{\mathrm{q}, 2}=0.016 \quad \delta G_{\mathrm{pq}, 2}=-0.006 \text {. }
$$

Estos valores indican que el proceso de concentración de la propiedad agraria que tuvo lugar en Guatemala entre 1964 y 1979 se debió, en primer lugar, a los cambios en los tamaños relativos, destacando la proliferación de las explotaciones más pequeñas. El otro componente significativo $\left(\delta G_{q, 2}\right.$ ) marca el efecto de las variaciones en las participaciones relativas, entre las que destaca el crecimiento del cuarto estrato. Es decir, la acumulación de la propiedad no tuvo lugar en la cúspide de la pirámide, sino en la clase estadística inmediatamente anterior.

Los resultados obtenidos deben interpretarse con cautela ya que dependen, en gran medida, de la disminución en la participación relativa del estrato más alto (pasó de 0.409 en 1950 a 0.260 en 1964, volviendo a caer a 0.227 en 1979) , y ésta puede ser producto de la reforma agraria, de modificaciones en las definiciones censales, o de errores en el levantamiento.

El pequeño valor relativo que asumió $\delta G_{p q, 2}$ (producto de las variaciones conjuntas) en ambos periodos, permite afirmar que esta distribución es poco sensible a la movilidad interestrato. Si este hecho se combina con la tendencia a la concentración inducida por los tamaños relativos en el periodo 1964-1979, debida, en gran medida, a la proliferación de los predios más pequeños (pasaron de 0.213 en 1964 a 0.411 en 1979), se puede sospechar que en ese lapso tuvo lugar un fuerte proceso de subdivisión de los predios chicos.

III.2 El cambio en la distribución del ingreso, en la población económicamente activa (PEA), en México, 1970 y 1980

Con los datos del cuadro 3 se preparó la información básica (cuadro 4) que permite calcular los componentes de la ecuación de descomposición (4).

El índice de concentración de Gini alcanzó, en 1970 y 1980 los siguientes valores:

$$
G_{1}=0.608 \text { y } G_{2}=0.557,
$$

donde los subíndices 1 y 2 representan los años 1970 y 1980, respectivamente. Esta información nos indica que en la década de los setenta tuvo 
CUADRO 3

Estructura del ingreso de la PEA por niveles salariales, 1970 y 1980 (relativos) ${ }^{15}$

\begin{tabular}{lccccc}
\hline & \multicolumn{2}{c}{1970} & & \multicolumn{2}{c}{1980} \\
\cline { 2 - 3 } \cline { 5 - 5 } Nivel de ingreso* & Prop. Pobl. & Porc. Inq. & & Prop. Pobl. & Porc. Inq. \\
\hline Sin ingresos & 0.103 & - & 0.236 & - \\
Menos de un sal. & 0.550 & 0.189 & 0.344 & 0.154 \\
1-menos de 2 & 0.219 & 0.237 & 0.248 & 0.314 \\
2-menos de 4 & 0.080 & 0.169 & 0.120 & 0.279 \\
4-menos de 6 & 0.023 & 0.081 & 0.033 & 0.140 \\
más de 6 sal. & 0.025 & 0.324 & 0.019 & 0.113 \\
\hline
\end{tabular}

* Los niveles de ingreso se elaboraron conforme al número de salarios mínimos. Para 1970 se tomó un salario mínimo general de 810 pesos y para 1980, de 4098.60 pesos. Fuentes: SPP, IX y X Censo General de Población, 1970 y 1980.

\section{CUADRO 4}

\section{Tamaños y participaciones relativas y sus cambios en el tiempo}

\begin{tabular}{ccccccc}
\hline Est. & $P_{1}$ & $q_{1}$ & $P_{2}$ & $q_{2}$ & $\delta p$ & $\delta q$ \\
\hline 1 & 0.103 & 0.000 & 0.236 & 0.000 & 0.133 & 0.000 \\
2 & 0.550 & 0.189 & 0.344 & 0.154 & -0.206 & -0.035 \\
3 & 0.219 & 0.237 & 0.248 & 0.314 & 0.029 & 0.077 \\
4 & 0.080 & 0.169 & 0.120 & 0.279 & 0.040 & 0.110 \\
5 & 0.023 & 0.081 & 0.033 & 0.140 & 0.010 & 0.059 \\
6 & 0.025 & 0.324 & 0.019 & 0.113 & -0.006 & -0.211 \\
\hline
\end{tabular}

Fuente: Con los datos del cuadro 3 se preparó la información básica.

lugar, en México, una tendencia hacia la equidistribución:

$$
\delta G=-0.051
$$

$\mathrm{Al}$ aplicar la descomposición se llega a:

$$
\delta G_{p}=-0.024 \quad \delta G_{q}=-0.012 \quad \delta G_{p q}=-0.018
$$

El resultado muestra que las tres componentes han actuado en el mismo sentido. Por una parte, el $\delta G_{p}$ es producto del crecimiento de los tamaños relativos de los estratos intermedios y de la disminución del estrato 2 (menos de un salario mínimo), aun cuando estos movimientos han

${ }^{15}$ Esta información fue tomada del artículo de Rosa Albina Gáravito y Fernando Gaona, "La distribución del ingreso en la PEA", El Cotidiano, número 3, diciembre, 1984-enero, 1985, Universidad Autónoma Metropolitana-Azcapotzalco. 
sido contrarrestados, en parte, por un crecimiento del estrato "sin ingresos". Por otra parte, $\delta G_{p q}$ (el segundo componente en importancia) da cuenta del hecho de que los procesos redistributivos implicaron movilidad interestrato, es decir, al aumentar la parte del pastel en los estratos inferiores, hubo personas que cambiaron de clase estadística, de manera que los estratos intermedios se nutrieron de los bajos y los altos. El tercer componente (el más débil), se debe a las variaciones en las participaciones relativas.

Hay que notar que en este ejemplo, a diferencia del anterior, el componente $\delta G_{p q}$ asumió un valor significativo. Esto se debe a que el criterio de clasificación es más sensible a variaciones en la distribución de la variable que en el caso de la distribución de la tierra en Guatemala.

\section{Conclusiones}

A partir de la idea de que detrás del cambio cronológico en el nivel de la desigualdad se pueden distinguir el (o los) proceso(s) que afecta(n) al tamaño relativo de las clases estadísticas y el (o los) proceso(s) que inciden sobre los parámetros de distribución de la variable, identificamos tres componentes que conforman la variación del índice de Gini. El primero $\left(\delta G_{q}\right)$, nos muestra aquella parte de la variación total que se debe a alteraciones en la repartición, bajo el supuesto que los tamaños relativos se mantienen constantes. El segundo $\left(\delta G_{p}\right)$, cuantifica la parte del cambio total en Gini debida a variaciones en los tamaños relativos, suponiendo constantes las participacipnes relativas. El tercero $\left(\delta G_{p q}\right)$ muestra el efecto combinado de los tamáños y participaciones relativas.

Esta descomposición, netamente descriptiva, abre las puertas para investigar los factores de que dependen los procesos identificados. La no separación de los componentes puede causar confusiones, ya que las determinantes de uno y otro suelen ser de naturaleza distinta. Esto implica, además, que el puro cálculo del cambio en el índice global puede llevar a conclusiones erróneas, debido a que es probable que esconda movimientos encontrados. En una aplicación particular puede darse el caso extremo de que la medida de Gini global $(G)$ no marque cambios en los niveles de desigualdad a pesar de haberse producido variaciones en los tamaños y participaciones relativas. Para ello basta con que sean de sentido opuesto y de la misma magnitud.

Hemos discutido las limitaciones en relación al sentido empírico o teórico del control estadístico del tamaño relativo como técnica que permite aislar el efecto de los cambios en las participaciones relativas sobre la medición de la desigualdad. La descomposición que ofrecemos puede utilizarse en aquellos casos en que el investigador decide construir sus intervalos de clase siguiendo criterios teóricos o bien identificando grupos 
sociales existentes en el dominio empírico.

Se ha señalado que el término $\delta G_{p q}$, puede ser un buen indicador de la sensibilidad de la distribución de frecuencias ante cambios en las participaciones relativas que desemboquen en movilidad interestrato. En los ejemplos de la sección III se ha mostrado la utilización de este concepto. En general, en las distribuciones "tipo Gini", es decir, aquellas en que la variable de clasificación es la misma que se reparte, se espera que la "sensibilidad estadística" sea mayor que en una distribución "tipo Levy" en la cual se mide la desigualdad de una variable sobre otra genérica, con la única condición de que ambas definan características de una misma unidad de registro.

Si bien nuestro interés se ha centrado en el caso particular del cambio en el tiempo o en el espacio del índice de Gini, el resultado a que hemos llegado es aplicable a cualquier función discreta $f[g(X, Y)]$ del grado total dos, como sería, por ejemplo, el índice entrópico de Theil. La descomposicón asume la forma:

$$
\delta f[g(X, Y]]=f\left[g\left(\delta X, Y_{0}\right)\right]+f\left[g\left(X_{0}, d Y\right)\right]+f[g[\delta X, d Y)],
$$

donde $X_{0}$ y $Y_{0}$ simbolizan los valores de las variables $X$ y $Y$ en el tiempo base o en la unidad espacial tomada como patrón de comparación. 